\title{
EVALUACIÓN DEL NIVEL DE DEGRADACIÓN DEL SUELO EN DOS SISTEMAS PRODUCTIVOS EN LA DEPRESIÓN DE QUÍBOR. II. CALIDAD DEL SUELO
}

\author{
Ingrid Acevedo ${ }^{1}$, Aymara Sánchez ${ }^{1}$ y Betty Mendoza ${ }^{1}$
}

RESUMEN

\begin{abstract}
En la zona hortícola de Quíbor, estado Lara, Venezuela, se ha aplicado como sistema de manejo convencional el cultivo de cebolla con riego por surcos, aunque actualmente se están implementando cambios en la producción agrícola que repercuten en el suelo, como es el cultivo de maíz super dulce regado por goteo. El objetivo de este trabajo fue comparar la calidad del suelo bajo el sistema convencional de ambos cultivos. La investigación fue de carácter cuasi-experimental, en la cual se tomaron muestras de suelo a tres profundidades en ambos sistemas de cultivo y se determinó la calidad en función del nivel de degradación de 13 atributos químicos, físicos y biológicos que habían sido seleccionados como indicadores en un trabajo previo. Tanto el suelo bajo el sistema convencional-maíz súper dulce como el convencional-cebolla presentaron baja calidad en los estratos estudiados particularmente porque compartieron alta degradación en la relación $\mathrm{Ca}$ /materia orgánica, macro/microporosidad y biomasa microbiana, aunque se diferenciaron en indicadores como el contenido de $\mathrm{P}$, la relación $\mathrm{C} / \mathrm{N}$ y $\mathrm{Ca} / \mathrm{Mg}$, la porosidad total y el cociente metabólico.

Palabras-clave adicionales: Cebolla, maíz súper dulce, manejo del suelo, propiedades del suelo
\end{abstract}

\begin{abstract}
Evaluation of the level of soil degradation in two productive systems in the Quibor depression. II. Soil quality In the horticultural zone of Quíbor, Lara state, Venezuela, the cultivation of onion with furrow irrigation has been applied as a conventional management system, although changes are currently being implemented in the agricultural production, that affect the soil, such as cropping of super sweet corn. The objective of this study was to compare, the soil quality under both production systems. The research was quasi-experimental in nature, with soil samplings at three different depths in each site. Soil quality was determined based on the level of degradation of 13 chemical, physical and biological attributes that had been selected as indicators in a previous work. Both the soil under the conventional system-super sweet corn and the conventional-onion system presented low quality in the studied strata, particularly because they shared high degradation in the ratios of $\mathrm{Ca} /$ organic matter and macro/microporosity, as well as in microbial biomass, although they differed in indicators such as $\mathrm{P}$ content, $\mathrm{C} / \mathrm{N}$ and $\mathrm{Ca} / \mathrm{Mg}$ ratios, total porosity and the metabolic ratio.
\end{abstract}

Additional keywords: Onion, soil management, soil properties, super sweet corn

\section{INTRODUCCIÓN}

El concepto de calidad del suelo surgió como una manera de evaluar en forma integral o parcial la capacidad de éste para cumplir diferentes funciones en forma sostenible (Karlen et al., 1997). Se puede definir como la aptitud del suelo para funcionar dentro de los límites ecológicos, sostener la productividad biológica, manteniendo la calidad ambiental, y promoviendo la salud de la flora y la fauna (Ochoa et al., 2007). Como no se puede medir directamente, para evaluar la calidad de un suelo se utilizan índices o indicadores basados en algunas características, propiedades o procesos de los suelos (García et al., 2012; Pla, 2013).

Dado que no existen criterios universales para evaluar los cambios en la calidad del suelo (Jiménez y González-Quiñones, 2006), numerosos investigadores utilizan el suelo bajo vegetación natural o no intervenido como nivel de referencia para establecer los indicadores (Rodríguez et al., 2009; Águila et al., 2016; Duval et al., 2016), por ser considerada la condición ideal y por presentar equilibrio en el ciclaje de los nutrientes (Navarrete

Recibido: Enero 3, 2020

Aceptado: Septiembre 28, 2020

${ }^{1}$ Dpto. Química y Suelos, Decanato de Agronomía, Universidad Centroccidental Lisandro Alvarado. Apdo. 400. Barquisimeto. Venezuela. e-mail: ingridacevedo@ucla.edu.ve (autor de correspondencia); aymaras@ucla.edu.ve; bmendoza2803@gmail.com 
et al., 2011). En otros estudios se han utilizado sitios de producción contrastante (alta y baja productividad) en diferentes tipos de suelo (Villarreal-Núñez et al., 2013), así como la selección de indicadores en sitios agrícolas y degradados de diferentes localidades y tipos de suelo (Estrada-Herrera et al., 2017). Además de evaluaciones de calidad, a nivel experimental, donde se contrastan diferentes tratamientos (Cairo et al., 2017). Por su parte, Vallejo et al. (2018) sugieren emplear un conjunto mínimo de datos que permita valorar la calidad de un suelo e integre una variedad de atributos que indiquen el estado y funcionamiento del mismo.

En este mismo sentido, los indicadores de calidad del suelo al no ser universales son elegidos en función del tipo de ambiente y suelo de la región en estudio (Cantú et al., 2007), dentro de un contexto de las características climáticas del sitio (INTA, 2000), o se utilizan indicadores previos establecidos en zona (Prieto-Méndez et al., 2013). Sin embargo, es importante verificar la aplicabilidad de los indicadores de calidad generados en una zona con respecto a suelos con diferentes condiciones de textura, manejo y uso.

El objetivo del presente estudio fue evaluar la calidad del suelo bajo el sistema convencional del cultivo de maíz súper dulce en la depresión de Quibor, Venezuela, mediante la comparación con los indicadores de calidad generados en el suelo bajo el manejo convencional-cebolla.

\section{MATERIALES Y MÉTODOS}

La investigación se realizó en la hacienda El Caujaral (serie de suelos Quíbor), ubicada en la depresión de Quíbor, municipio Jiménez, estado Lara, Venezuela, con altitud de 600 msnm, precipitación de 400 a $500 \mathrm{~mm}$ anuales y temperatura media de $25,5{ }^{\circ} \mathrm{C}$. El suelo está descrito como un Typic Haplocambids arcilloso, isohipertérmico, mixto, calcáreo. Se estudiaron dos sistemas productivos: convencional-cebolla (suelo arcillo-limoso y 16 años de utilización), y convencional-maíz súper dulce (suelo franco arcillo-limoso, y menos de 5 años de cambio de uso, anteriormente bajo el sistema convencionalcebolla).

Debido a lo anterior y al hecho de que ambos sistemas presentaron un historial de manejo en la misma trayectoria, lo cual representa una condición estándar para estimar la calidad del suelo (INTA, 2000), el sistema convencional-cebolla se consideró como el sistema de referencia.

Se realizaron muestreos en ambos suelos a profundidades de $0-5 \mathrm{~cm}, 5-18 \mathrm{~cm}$ y $18-50 \mathrm{~cm}$ y se evaluaron atributos que, en un ensayo previo (Acevedo et al., 2021), resultaron seleccionados como indicadores de calidad del suelo. Los atributos fueron de tipo físico (porosidad total, relación macro/ microporosidad e índice de separabilidad de partículas), químico ( $\mathrm{pH}$, nitrógeno total, relación $\mathrm{C} / \mathrm{N}$, fósforo, y las relaciones $\mathrm{Ca} / \mathrm{K}, \mathrm{Ca} / \mathrm{Mg}, \mathrm{Ca} /$ materia orgánica $\mathrm{y}$ carbono orgánico/arcilla) y biológico (respiración basal y carbono microbiano).

Todos los detalles referentes a los sistemas productivos, muestreo de suelos y determinación de las variables fueron reportados en una publicación previa (Acevedo et al., 2021).

Para evaluar la calidad del suelo, a partir de los indicadores mencionados, se manejaron cinco categorías con base en SCS-USDA (1983), Romig et al. (1995) y Rodríguez et al. (2009), y se utilizaron los valores críticos referenciados por Torres et al. (2006) y Mendoza y Florentino (2011) (Cuadro 1).

Se estimó el valor del índice de degradación (ID) mediante la sumatoria de la clase de degradación de los indicadores (químicos, físicos y biológicos) entre la sumatoria de la clase máxima; además de la calidad del suelo (ICS) por el inverso del ID. Con base en ID e ICS, se estimó la calidad del suelo de acuerdo a las clases establecidas en el Cuadro 2.

\section{RESULTADOS Y DISCUSIÓN}

\section{Degradación de los suelos}

Indicadores químicos. Los indicadores químicos que reflejaron la mayor degradación diferencial entre los suelos con diferentes manejos y usos fueron el contenido de fósforo y la relación $\mathrm{Ca} / \mathrm{Mg}$, con menores valores en el suelo del sistema convencional cebolla (Cuadro 3), lo cual se atribuye al manejo aplicado en el mismo, donde se empleó doble fertilización fosfórica respecto al suelo bajo el sistema con maíz súper dulce, el cual presentó niveles medios de $\mathrm{P}$ (Havlin et al., 2016). La depresión de Quíbor se caracteriza por contenidos bajos de P (Torres et al., 2017). Con respecto a la relación $\mathrm{Ca} / \mathrm{Mg}$, ésta reflejó muy alta 
degradación en el suelo bajo el sistema con maíz súper dulce, lo cual indica un desbalance entre estos elementos que restringen la adsorción de magnesio, atribuido al alto aporte de potasio en detrimento de la disponibilidad de magnesio, a pesar de la aplicación del mismo en la fertilización. El suelo presentó niveles bajos de $\mathrm{Mg}$ (112,7 a $135,3 \mathrm{mg} \mathrm{kg}^{-1}$ ) y muy altos de Ca de 4400 a 4889,3 $\mathrm{mg} \mathrm{kg}^{-1}$ (Havlin et al., 2016), lo que ocasiona la alta relación $\mathrm{Ca} / \mathrm{Mg}$.

Cuadro 1. Valores críticos para establecer la clase de degradación por los indicadores químicos, físicos y biológicos de calidad del suelo*

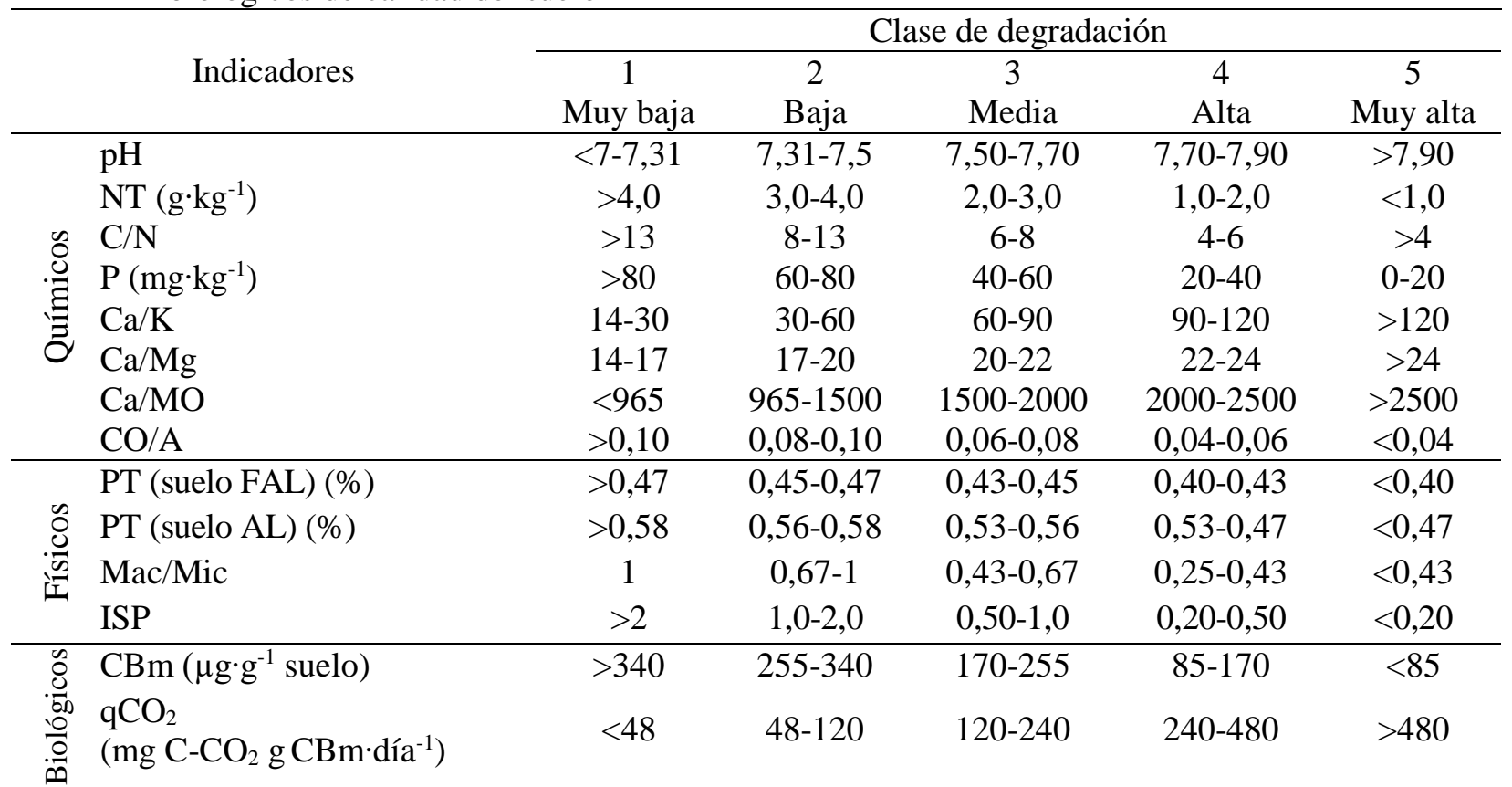

F: franco; AL: arcillo limoso; NT: Nitrógeno total; C/N: Relación carbono/nitrógeno; P: Fósforo; Ca/Mg: Relación calcio/ Magnesio; $\mathrm{Ca} / \mathrm{K}$ : Relación Calcio/Potasio; Ca/MO: Relación Calcio/Materia orgánica; ISP: Índice de separabilidad de partículas; CO/A: Relación carbono orgánico/Arcilla; $\mathrm{CBm}$ : Carbono de la biomasa microbiana; $\mathrm{qCO}_{2}$ : Cociente metabólico. *Tomado de Torres et al. (2006); Mendoza y Florentino (2011)

Cuadro 2. Valores de índices de degradación y calidad de suelo*

\begin{tabular}{ccccc}
\hline $\begin{array}{c}\text { Índice de } \\
\text { degradación }\end{array}$ & $\begin{array}{c}\text { Intensidad de } \\
\text { degradación }\end{array}$ & $\begin{array}{c}\text { Índice de calidad } \\
\text { del suelo }\end{array}$ & $\begin{array}{c}\text { Calidad del } \\
\text { suelo }\end{array}$ & Clase \\
\hline$<0,20$ & Muy baja & $>5,00$ & Muy alta & 1 \\
$0,20-0,40$ & Baja & $5,00-2,50$ & Alta & 2 \\
$0,40-0,60$ & Media & $2,50-1,67$ & Media & 3 \\
$0,60-0,80$ & Alta & $1,67-1,25$ & Baja & 4 \\
$0,80-1,00$ & Muy alta & $1,25-1,00$ & Muy baja & 5 \\
\hline
\end{tabular}

*Tomado de Mendoza y Florentino (2011)

Otro indicador que presentó una clase de degradación contrastante entre ambos suelos fue la relación $\mathrm{C} / \mathrm{N}$, con degradación muy alta en el suelo bajo el sistema con cebolla, en comparación al suelo bajo el sistema con maíz súper dulce el cual presentó degradación media. A pesar de que ambos suelos presentaron baja relación $\mathrm{C} / \mathrm{N}$, la alta degradación en el sistema con cebolla se atribuye a una explotación intensiva del suelo que originaría una mineralización acelerada, o un exceso de nitrógeno amoniacal. La relación $\mathrm{C} / \mathrm{N}$ ideal, que favorece la actividad microbiana, corresponde a valores de 8,5 a 10 (Fuentes, 1999). Por su parte, la degradación de la relación $\mathrm{Ca} / \mathrm{MO}$ fue muy alta en ambos suelos

El indicador $\mathrm{pH}$ reflejó una clase de 
degradación ligeramente menor en el suelo del sistema con maíz súper dulce, lo cual se atribuye a las aplicaciones de ácido nítrico en el fertiriego y al uso de fertilizantes de reacción ácida como el fosfato monoamónico, que influyen en la disminución del $\mathrm{pH}$.

Al comparar la clase de degradación entre las diferentes profundidades en el suelo, solo se presentó un comportamiento diferencial favorable en el primer estrato $(0-5 \mathrm{~cm})$ para los indicadores $\mathrm{CO} / \mathrm{A}$ y $\mathrm{Ca} / \mathrm{K}$ en el suelo con maíz súper dulce. Este último puede estar asociado al aporte de potasio en la fertilización, lo cual mantiene una relación que favorece la disponibilidad de este nutriente para las plantas. Similarmente, Jaurixje et al. (2013) encontraron valores altos de potasio en la zona de Quíbor relacionados con la aplicación de fertilizante potásico.

Cuadro 3. Clase de degradación de los suelos con los sistemas convencional-cebolla y maíz súper dulce en función de sus indicadores químicos

\begin{tabular}{|c|c|c|c|c|c|}
\hline \multirow{2}{*}{ Indicador } & \multirow{2}{*}{ Estrato } & \multicolumn{2}{|c|}{ Cebolla } & \multicolumn{2}{|c|}{ Maíz súper dulce } \\
\hline & & Promedio & Clase & Promedio & Clase \\
\hline \multirow{3}{*}{$\mathrm{pH}$} & 1 & 7,65 & 3 & 7,50 & 2 \\
\hline & 2 & 7,57 & 3 & 7,41 & 2 \\
\hline & 3 & 7,52 & 3 & 7,37 & 2 \\
\hline \multirow{3}{*}{ Nitrógeno total $\left(\mathrm{g} \cdot \mathrm{kg}^{-1}\right)$} & 1 & 2,9 & 3 & 1,35 & 4 \\
\hline & 2 & 2,9 & 3 & 1,25 & 4 \\
\hline & 3 & 2,9 & 3 & 1,26 & 4 \\
\hline \multirow{3}{*}{ Carbono/nitrógeno $(\mathrm{C} / \mathrm{N})$} & 1 & 3,44 & 5 & 6,38 & 3 \\
\hline & 2 & 3,44 & 5 & 7,23 & 3 \\
\hline & 3 & 3,24 & 5 & 6,22 & 3 \\
\hline \multirow{3}{*}{ Fósforo $\left(\mathrm{mg} \cdot \mathrm{kg}^{-1}\right)$} & 1 & 126 & 1 & 33 & 4 \\
\hline & 2 & 145 & 1 & 31 & 4 \\
\hline & 3 & 111 & 1 & 24 & 4 \\
\hline \multirow{3}{*}{ Calcio/magnesio (Ca/Mg) } & 1 & 14,26 & 1 & 33,47 & 5 \\
\hline & 2 & 15,48 & 1 & 42,01 & 5 \\
\hline & 3 & 16,30 & 1 & 40,48 & 5 \\
\hline \multirow{3}{*}{ Calcio/potasio $(\mathrm{Ca} / \mathrm{K})$} & 1 & 48,42 & 2 & 27,18 & 1 \\
\hline & 2 & 42,53 & 2 & 40,45 & 2 \\
\hline & 3 & 52,80 & 2 & 50,79 & 2 \\
\hline \multirow{3}{*}{ Calcio/materia orgánica $(\mathrm{Ca} / \mathrm{MO})$} & 1 & 3013,71 & 5 & 3133,3 & 5 \\
\hline & 2 & 3004,08 & 5 & 3116,9 & 5 \\
\hline & 3 & 3264,66 & 5 & 3622,8 & 5 \\
\hline \multirow{3}{*}{ Carbono orgánico/ arcilla (CO/A) } & 1 & 0,02 & 5 & 0,061 & 3 \\
\hline & 2 & 0,02 & 5 & 0,056 & 4 \\
\hline & 3 & 0,02 & 5 & 0,053 & 4 \\
\hline \multirow{3}{*}{$\Sigma$ clase/clase máxima } & 1 & & $25 / 40$ & & $27 / 40$ \\
\hline & 2 & & $25 / 40$ & & $29 / 40$ \\
\hline & 3 & & $25 / 40$ & & $29 / 40$ \\
\hline
\end{tabular}

Clase de degradación: 1) muy baja; 2) baja; 3) media; 4 alta y 5) muy alta. Estratos: 1) 0-5 cm;2) 5-18 cm;3) $18-50 \mathrm{~cm}$

Indicadores físicos. Los indicadores físicos que reflejaron mayor degradación diferencial entre los suelos fueron la porosidad total y el índice de separabilidad de partículas (Cuadro 4), características que se vinculan con la textura de los suelos y no a su manejo. A partir de estos indicadores se infiere que el suelo del sistema con maíz súper dulce presenta riesgo a la formación de costras y compactación por el bajo valor del ISP que genera baja estabilidad estructural. Valores menores a 0,5 se consideran inestables (Pulido et al., 2009), condición que predomina en los suelos de la serie Quíbor (Reyes y Willians, 2010).

La clase de degradación de los suelos no varió 
con la profundidad en el sistema productivo con maíz súper dulce, pero se incrementó en el sistema con cebolla en los indicadores de PT y Mac/Mic, lo que pudiera sugerir restricciones por compactación y se debería atribuir a un mal manejo del suelo.

Indicadores biológicos. El indicador que reflejó mayor degradación diferencial entre los suelos fue el cociente metabólico, y el suelo que presentó mayor degradación, con valores altos del $\mathrm{qCO}_{2}$ fue el sistema con cebolla (Cuadro 5). La baja eficiencia metabólica sugiere que los microorganismos del suelo se encontrarían bajo estrés. Paolini (2017) señala que en suelos con elevadas cantidades de compuestos fácilmente disponibles y degradables este cociente se incrementa, por lo que nuestros resultados podrían estar asociados al aporte de materia orgánica al suelo. Valores altos de la respiración basal indican mayor actividad microbiana en este sistema, imputables a la adición permanente y continua de fuentes exógenas de materia orgánica lábil y la subsecuente estimulación de los microorganismos heterotróficos (Okur et al., 2015).

La clase de degradación de los suelos no varió con la profundidad en el sistema productivo con maíz súper dulce, pero en el sistema con cebolla disminuyó ligeramente con la profundidad, al considerar la biomasa microbiana, porque esta responde tempranamente a cambios en las condiciones del suelo (Navarrete et al., 2011), que pueden ser ocasionados por efectos de compactación. Además, la respuesta se podría atribuir a la normal variabilidad espacial del suelo.

Cuadro 4. Clase de degradación de los suelos con el sistema convencional-cebolla y maíz súper dulce en función de sus indicadores físicos

\begin{tabular}{|c|c|c|c|c|c|}
\hline \multirow[t]{2}{*}{ Indicadores } & \multirow[t]{2}{*}{ Estratos } & \multicolumn{2}{|c|}{ Convencional-cebolla } & \multicolumn{2}{|c|}{$\begin{array}{l}\text { Convencional-maíz } \\
\text { súper dulce }\end{array}$} \\
\hline & & Promedio & Nivel & Promedio & Nivel \\
\hline \multirow{3}{*}{ Porosidad total (\%) } & 1 & 55,25 & 3 & 49,48 & 1 \\
\hline & 2 & 51,57 & 4 & 47,78 & 1 \\
\hline & 3 & 48,70 & 4 & 47,09 & 1 \\
\hline \multirow{3}{*}{ Macroporos/microporos } & 1 & 0,45 & 3 & 0,39 & 4 \\
\hline & 2 & 0,28 & 4 & 0,33 & 4 \\
\hline & 3 & 0,20 & 5 & 0,36 & 4 \\
\hline \multirow{3}{*}{$\begin{array}{l}\text { Índice de separabilidad de } \\
\text { partículas (ISP) }\end{array}$} & 1 & 0,94 & 3 & 0,18 & 5 \\
\hline & 2 & 0,94 & 3 & 0,21 & 4 \\
\hline & 3 & 0,96 & 3 & 0,19 & 5 \\
\hline \multirow{3}{*}{$\Sigma$ clase/clase máxima } & 1 & & $9 / 15$ & & $10 / 15$ \\
\hline & 2 & & $11 / 15$ & & $9 / 15$ \\
\hline & 3 & & $12 / 15$ & & $10 / 15$ \\
\hline
\end{tabular}

Clase de degradación: 1) muy baja; 2) baja; 3) media; 4 alta y 5) muy alta. Estratos: 1) 0-5 cm;2) 5-18 cm; 3) 18-50 cm

Calidad de los suelos. Con base en los indicadores químicos, físicos y biológicos, los suelos bajo estudio presentaron similar intensidad de la degradación y calidad en los tres estratos de profundidad estudiados (Cuadro 6). En general, ambos suelos presentan alta degradación y baja calidad, a pesar de las diferencias en varios indicadores químicos, físicos y biológicos. En una investigación similar, Mendoza et al. (2014), haciendo uso de un mayor número de indicadores, también reportaron baja calidad del suelo con el manejo convencional de cebolla, lo cual está relacionado con la muy baja relación $\mathrm{C} / \mathrm{N}$, baja
$\mathrm{CO} / \mathrm{A}$ y alta $\mathrm{Ca} / \mathrm{MO}$, y sugieren la necesidad de aplicaciones de MO más estable. En nuestro caso, se encontró que en un estrato del suelo cultivado con cebolla se presentan problemas de compactación y restricciones en el crecimiento de la biomasa microbiana. Las propiedades de los suelos pueden ser muy heterogéneas en función de la variabilidad del manejo del cultivo establecido (Saavedra et al., 2019).

La baja calidad del suelo con el sistema productivo convencional-maíz súper dulce está relacionada a los niveles bajos de nitrógeno total y fósforo disponible, y alta relación $\mathrm{Ca} / \mathrm{Mg}$ y $\mathrm{Ca} / \mathrm{MO}$, 
producto de las condiciones intrínsecas del suelo y del plan de fertilización utilizado. Además de las limitaciones edáficas afines con la textura del suelo (Reyes y Willians, 2010). Asimismo, se relaciona con los valores bajos de biomasa microbiana y alto índice metabólico debido a la baja eficiencia de los microorganismos del suelo. Asimismo, el deterioro de las condiciones físicas del suelo pudiera estar relacionadas con las restricciones en el desarrollo de microorganismos (Jaurixje et al., 2013).

Cuadro 5. Clase de degradación de los suelos con el sistema convencional-cebolla y maíz súper dulce en función de sus indicadores biológicos

\begin{tabular}{|c|c|c|c|c|c|}
\hline \multirow[t]{2}{*}{ Indicadores } & \multirow[t]{2}{*}{ Estratos } & \multicolumn{2}{|c|}{ Convencional-cebolla } & \multicolumn{2}{|c|}{$\begin{array}{l}\text { Convencional-maíz } \\
\text { súper dulce }\end{array}$} \\
\hline & & Promedio & Nivel & Promedio & Nivel \\
\hline \multirow{2}{*}{$\mathrm{CBm}\left(\mu \mathrm{g} \mathrm{CBm} \cdot \mathrm{g}^{-1}\right.$ suelo $)$} & 1 & 83,49 & 5 & 150 & 4 \\
\hline & 2 & 86,56 & 4 & 134 & 4 \\
\hline \multirow{2}{*}{$\begin{array}{l}\text { Cociente metabólico (mg C- } \\
\left.\mathrm{CO}_{2} \cdot \mathrm{g}^{-1} \mathrm{CBm} \mathrm{día}{ }^{-1}\right)\end{array}$} & 1 & 552,48 & 5 & 234,7 & 3 \\
\hline & 2 & 649,44 & 5 & 224,4 & 3 \\
\hline \multirow{2}{*}{$\Sigma$ clase/clase máxima } & 1 & & $10 / 10$ & & $7 / 10$ \\
\hline & 2 & & $9 / 10$ & & $7 / 10$ \\
\hline
\end{tabular}

CBm: Carbono de la biomasa microbiana. Clase de degradación: 1) muy baja; 2) baja; 3) media; 4) alta; 5) muy alta. Estratos: 1) $0-5 \mathrm{~cm}$; 2) $5-18 \mathrm{~cm}$; 3) $18-50 \mathrm{~cm}$

Cuadro 6. Calidad de los suelos bajo el sistema convencional-cebolla y maíz súper dulce, en función de sus indicadores químicos, físicos y biológicos en general

\begin{tabular}{lccc}
\hline Variables & Estratos & Cebolla & Maíz súper dulce \\
\hline \multirow{2}{*}{$\Sigma$ clase total/ $\Sigma$ clase máxima total } & 1 & $44 / 65$ & $44 / 65$ \\
& 2 & $45 / 65$ & $45 / 65$ \\
& 3 & $37 / 55$ & $39 / 55$ \\
\hline \multirow{2}{*}{ Índice de degradación (intensidad) } & 1 & 0,68 (alta) & 0,68 (alta) \\
& 2 & 0,69 (alta) & 0,69 (alta) \\
Índice de calidad (calidad del suelo) & 3 & 0,67 (alta) & 0,71 (alta) \\
& 1 & 1,48 (baja) & 1,48 (baja) \\
& 2 & 1,44 (baja) & 1,44 (baja) \\
\hline
\end{tabular}

Îndice de degradación: $\Sigma$ clase total/ $\Sigma$ clase máxima total. Calidad del suelo: 1) muy alta; 2) alta; 3) media; 4) baja; 5) muy baja. Estratos: 1) $0-5 \mathrm{~cm}$;) $5-18 \mathrm{~cm}$; 3$) 18-50 \mathrm{~cm}$

\section{CONCLUSIONES}

Los atributos químicos, físicos y biológicos seleccionados como indicadores reflejaron condiciones de degradación en cada suelo, relacionadas a características intrínsecas y de manejo.

Tanto el suelo bajo el sistema convencionalmaíz súper dulce como el del sistema convencional-cebolla presentaron baja calidad, a pesar de mostrar entre ellos diferencias en el balance de nutrientes, así como limitaciones físicas y biológicas. Por ello, es recomendable incrementar la fertilización de $\mathrm{P}$ y $\mathrm{Mg}$, así como realizar aplicación de MO que favorezca la estabilidad del suelo y la biomasa microbiana.

\section{LITERATURA CITADA}

1. Acevedo, I., A, Sánchez y B. Mendoza. 2021. Evaluación del nivel de degradación del suelo en dos sistemas productivos en la Depresión de Quíbor. I. Análisis multivariado. Bioagro 33(1): 59-66.

2. Águila, E., Y. Marrero, H. Hernández y Y. Ruiz. 2016. Efecto del uso del suelo sobre su calidad en áreas de la Finca "Baños de Marrero". Centro Agrícola 43(2): 14-22. 
3. Cairo, P., J. Machado, O. Rodríguez y A. Rodríguez. 2017. Efecto de abonos órganominerales sobre la calidad del suelo, impacto en el rendimiento de la caña de azúcar. Centro Azúcar 44(4): 12-20.

4. Cantú, M., A. Becker; J. Bedano y H. Schiavo. 2007. Evaluación de la calidad de suelos mediante el uso de indicadores e índices. CI. Suelo (Argentina) 25(2): 173-178.

5. Duval, M., J. Galantini; J. Martínez y J. Iglesias. 2016. Comparación de índices de calidad de suelos agrícolas y naturales basados en el carbono orgánico. Ciencia del suelo (Argentina) 34(2): 197-209.

6. Estrada-Herrera, I., C. Hidalgo-Moreno, R. Guzmán-Plazola, J. Almaraz, H. NavarroGarza, J. Etchevers-Barra. 2017. Indicadores de calidad de suelo para evaluar su fertilidad. Agrociencia 51: 813-831.

7. Fuentes, J. 1999. El Suelo y los Fertilizantes. Ediciones Mundi-Prensa. Madrid.

8. García, Y., W. Ramírez y S. Sánchez. 2012. Indicadores de la calidad de los suelos: una nueva manera de evaluar este recurso. Pastos y Forrajes, 35 (2): 125-138.

9. Havlin, J., S.L. Tisdale, W.L. Nelson y J.D. Beaton. 2016. Soil Fertility and Fertilizers. Pearson Publish., London

10.INTA (Instituto Nacional de Tecnología Agropecuaria). 2000. Guía para la Evaluación de la Calidad y Salud del Suelo (traducción de A. Luters y J. Salazar). Cartografía de Suelos y Evaluación de Tierras. INTA, Buenos Aires.

11.Jaurixje, M., D. Torres, B. Mendoza, M. Henríquez y J. Contreras. 2013. Propiedades físicas y químicas del suelo y su relación con la actividad biológica bajo diferentes manejos en la zona de Quíbor, Estado Lara. Bioagro, 25 (1): 47-56.

12.Jiménez, B. y V. González-Quiñones. 2006. La calidad de suelos como medida para su conservación. Edafología, 13 (3): 125-138.

13.Karlen, D. L., Mausbach, M. J., Doran, J. W., Cline, R. G., Harris, R. R, and Schuman, G. E. 1997. Soil quality: a concept, definition, and framework for evaluation. Soil Sci. Soc. Am. J. 61: 4-10.

14.Mendoza, B. y A. Florentino. 2011. Calidad física, química y biológica de dos suelos bajo diferentes sistemas de uso y manejo en Quíbor, estado Lara. Memoria de Congreso Nacional de Ciencias del suelo. www.sian.inia.gob.ve/ repositorio/congresos/CVCS19/uso_manejo_su elo/UMS31 (consulta de septiembre 24, 2019).

15. Mendoza, B., A. Florentino, M. Henríquez y O. Rodríguez. 2014. Atributos biológicos de dos suelos de Quíbor bajo diferentes uso y manejo. Rev. Fac. Agron. (LUZ) Supl. 1: 644-655.

16. Navarrete, A., G. Vela, J. López, M. Rodríguez. 2011. Naturaleza y utilidad de los indicadores de calidad del suelo. ContactoS 80: 29-37.

17. Ochoa, V., B. Hinojosa, B. Gómez-Muñoz y R. García-Ruiz. 2007. Actividades enzimáticas como indicadores de calidad del suelo en agroecosistemas ecológicos. Ini Inv. 2: i-x.

18. Okur, N., H. H. Kayikcioglu, F. Ates, and B. Yagmur. 2015. A comparison of soil quality and yield parameters under organic and conventional vineyard systems in Mediterranean conditions (West Turkey). Biol. Agric. Hortic. 32: 1-12.

19.Paolini, J. E. 2017. Actividad microbiológica y biomasa microbiana en suelos cafetaleros de los Andes venezolanos. Terra Latinoamericana 36: 13-22.

20.Pla-Sentis, I. 2013. Aproximaciones empíricas para la evaluación de la calidad del suelo: ventajas e inconvenientes. XX Congreso Venezolano de la Ciencia del Suelo. San Juan de los Morros. SIAN.

21.Prieto-Méndez, J., F. Prieto-García, O. AcevedoSandoval, M. Méndez-Marzo. 2013. Indicadores e índices de calidad de los suelos (ICS) cebaderos del sur del estado de Hidalgo, México. Agronomía Mesoamericana 24(1): 83-91.

22.Pulido-Moncada, M.A., D. Lobo-Luján y Z. Lozano-Pérez. 2009. Asociación entre indicadores de estabilidad estructural y la materia orgánica en suelos agrícolas de Venezuela. Agrociencia 43(3): 221-230.

23. Reyes R, y J. Willians. 2010. Evaluación de la susceptibilidad a la compactación en cuatro series de suelo bajo uso agrícola en Venezuela. 2010. Bioagro 22(1): 29-36.

24.Rodríguez, N., A. Florentino, D. Torres, H. Yendis y F. Zamora. 2009. Selección de 
indicadores de calidad de suelo en tres tipos de uso de la tierra en la planicie de Coro estado Falcón. Rev. Fac. Agron. (LUZ) 26: 340-341.

25.Romig, D.E., M.J. Garlynd, R.F. Harris y K. McSweeney. 1995. How farmers assess soil health and quality. J. Soil and Water Conservation 50(3): 229-236.

26.Saavedra-Mora, D., V. Murcia-Torrejano, L. Machado-Cuellar, J. Sánchez-Cerquera, L.F. Estrada-Quintero y C.M. Ordoñez-Espinosa. 2019. Propiedades físicas y químicas de suelos y su relación con sistemas de producción en el municipio Campoalegre, departamento del Huila, Colombia. Bioagro 31(2): 151-158.

27.SCS-USDA (Soils Conservation Service). 1983. National Soils-Handbook. Washington, D.C.

28.Torres, D., A. Florentino y M. López. 2006. Indicadores e índices de calidad del suelo en un
Ultisol bajo diferentes prácticas de manejo conservacionista en Guárico, Venezuela. Bioagro 18(2): 83-91.

29. Torres, D., J. Álvarez, J. Contreras, M. Henríquez, W. Hernández, J. Lorbes y J. Mogollón. 2017. Identificación de potencialidades y limitaciones de suelos agrícolas del estado Lara, Venezuela. Bioagro 29(3): 207-218.

30.Vallejo, V., L. Afanador, M. Hernández y D. Parra. 2018. Efecto de la implementación de diferentes sistemas agrícolas sobre la calidad del suelo en el municipio de Cachipay, Cundinamarca, Colombia. Bioagro 30(1): 27-38.

31.Villarreal-Núñez, J., I. Pla-Sentis, L. AgudoMartínez, J. Villaláz-Perez, F. Rosales y L. Pocasangre. 2013. Índice de calidad del suelo en áreas cultivadas con banano en Panamá. Agronomía Mesoamericana 24(2): 301-315. 